\title{
Increased cerebral blood flow velocities in newborn infants of smoking mothers
}

\author{
H. Abdul-Khaliq, H. Segerer, W. Luck, M. Obladen \\ Department of Neonatology, Children's Hospital, Universitätsklinikum Rudolf Virchow, \\ Free University, Heubnerweg 6, W-1000 Berlin 19
}

Received April 15, 1992 / Accepted July 17, 1992

\begin{abstract}
Cerebral blood flow velocities (CBFV) were measured by the pulsed Doppler method in 41 infants of smoking mothers and in 59 apparently healthy control infants. Although gestational age, birth weight, and systolic blood pressure were lower in infants exposed to tobacco smoke prenatally, systolic (65 \pm 11 vs. $47 \pm 12 \mathrm{~cm} / \mathrm{s}$, mean $\pm \mathrm{SD} ; P<0.001)$, mean $(36 \pm 6$ vs. $25 \pm 6 \mathrm{~cm} / \mathrm{s}$; $P<0.001)$, and diastolic $(17 \pm 4$ vs $13 \pm 4 \mathrm{~cm} / \mathrm{s} ; P<0.001)$ CBFVs in the anterior cerebral artery were significantly higher when compared to control infants. Similar differences were seen in the internal carotid and in the basilar arteries. Multiple regression analysis did not reveal differences other than maternal smoking to explain these observations. We conclude that prenatal tobacco smoke exposure is related to increased CBFVs in newborn infants. Further studies should determine whether this relation is not only statistical but causal and whether increased CBFVs are an indicator of prolonged effects of prenatal tobacco smoke exposure.
\end{abstract}

Key words: Cerebral arteries - Blood flow velocity Newborn infant - Tobacco smoke - Pregnancy

\section{Introduction}

In Western countries, $25 \%-30 \%$ of pregnant women smoke [5, 21]. Effects of maternal smoking on fetal and neonatal morbidity and mortality are well established [5, 13, 21]. Moreover, prenatal exposure to tobacco smoke has been shown to be related to sudden infant death [4, $5]$ and to impaired mental development [15, 19, 20]. Maternal smoking has been demonstrated to increase fetal blood flow velocities in the descending thoracic aorta and in umbilical vessels [10,14]. Ahlsten et al. [1] showed an impaired vascular reactivity of the skin in newborn infants of smoking mothers $24-48 \mathrm{~h}$ after birth. Similar ef-

Correspondence to: $\mathrm{H}$. Segerer

Abbreviations: $\mathrm{BP}=$ blood pressure $\mathrm{CBFV}=$ cerebral blood flow velocity fects on cerebral vessels have not been demonstrated but it has been reported that maternal drug addiction [3] is related to changes of cerebral blood flow velocities (CBFVs) in neonates.

As we wanted to investigate whether postnatal CBFVs are also influenced by prenatal tobacco smoke exposure, we measured CBFVs by Doppler sonography in infants who were born to smoking mothers, and in healthy newborns of non-smoking mothers.

\section{Methods}

Between January and May 1991, 41 consecutive infants born to mothers who declared smoking ten or more cigarettes per day during their pregnancy were studied. In each case, the mother was asked about smoking habits, drug abuse, and medications. We excluded infants who presented with factors that are known or suspected to influence CBFVs: immaturity with less than $35 \mathrm{com}$ pleted weeks of gestation, birth weight below 10th percentile, mean arterial blood pressure $<30 \mathrm{~mm} \mathrm{Hg}$ during the 1 st day of life, perinatal asphyxia with an umbilical artery $\mathrm{pH}<7.15$, intra- or periventricular haemorrhage, signs of persistent ductus arteriosus, venous haematocrit $>65 \%$, infants of mothers suffering from diabetes, gestosis, hypertension with blood pressures $>160 / 100 \mathrm{~mm} \mathrm{Hg}$ during pregnancy, or drug abuse. Informed parental consent was obtained for the Doppler examination and urine collection.

Fifty-nine healthy newborn infants born to non-smoking mothers in the same time period served as controls after informed parental consent had been obtained. The same exclusion criteria were applied to these infants.

At examination, all infants were between 20 and $42 \mathrm{~h}$ old. The Doppler examination was postponed within this time range if the infant was not quiet during the first visit. Blood pressure (BP) was measured at the time of the Doppler examination using a Dinamap (Criticon Inc., Florida, USA). A pulsed-Doppler, two-dimensional ultrasound scanner (Ultramark 4, Advanced Technology Laboratories, Inc., Solingen, FRG) with a $5 \mathrm{MHz}$ Doppler probe was used to exclude structural abnormalities and to measure CBFVs. All Doppler examinations were carried out by the same investigator (H. A-K.). CBFVs were examined in the internal carotid artery immediately beneath the lateral edge of the sella turcica, in the anterior cerebral artery directly anterior to the third ventricle beneath the lower edge of the corpus callosum, and in the basilar artery between pons and the skull base [7]. Doppler frequencies were recorded when the sharpest characteristic visual and highest audible signals were obtained [16]. A tracing of ten equal heart cycles was printed out (Mitsubishi Video Copy Processor K 70S, Mit- 
subishi Electric Corporation, Japan). Maximal systolic, mean, and end-diastolic velocities could be read after internal computing of the Doppler frequencies.

Urinary cotinine excretion was used to confirm or exclude prenatal tobacco smoke exposure in the studied infants. Urine collection was commenced immediately after the Doppler examination for $6 \mathrm{~h}$. Samples were frozen at $-20^{\circ} \mathrm{C}$ until time of analysis. Cotinine concentrations were measured by gas liquid chromatography as described previously [12]. Urinary cotinine excretion was determined as cotinine/creatinine ratio ( $\mathrm{ng} / \mathrm{mg})$.

The Mann-Whitney U-test was applied to evaluate differences between variables of smoke exposed and control infants. In an attempt to create a model that allows prediction of each infant's CBFVs on the basis of factors which could theoretically influence them, we included the following factors into a multiple regression analysis: prenatal tobacco smoke exposure, birth weight, gestational age, umbilical artery $\mathrm{pH}$; age, heart rate, and systolic $\mathrm{BP}$ at examination. All calculations were done with the SPSS-PC+ statistical package (SPSS Inc., Chicago, USA). A $P$ value of $<0.05$ was considered to be statistically significant.

\section{Results}

Mean gestational age ( $38.8 \pm 1.6$ vs. $39.6 \pm 1.0$ weeks; mean $\pm \mathrm{SD} ; P<0.01)$, mean birth weight $(3147 \pm 469$ vs. $3402 \pm 134 \mathrm{~g} ; P<0.01)$, and systolic BP $(67.7 \pm 10.8$ vs. $71.8 \pm 11.3, P<0.05$ ) were significantly lower in infants exposed to cigarette smoke before birth. There were no significant differences in umbilical artery $\mathrm{pH}$, Apgar scores at 1, 5 or $10 \mathrm{~min}$, heart rate, diastolic or mean BP, and postnatal age at examination. None of the 100 infants studied had to be referred to a special care nursery.

In each of the three cerebral arteries examined, maximal systolic, mean, and diastolic flow velocities were significantly higher in infants with maternal smoking history than in control infants (Table 1).

In the group of babies born to smoking mothers, urine samples could be obtained from 33 infants. In 3 of these, urinary cotinine concentrations were below the detection limit $(5 \mathrm{ng} / \mathrm{ml})$. In the remaining 30 infants, cotinine excretion ranged from 20 to 3126 , median $193.3 \mathrm{ng} /$

Table 1. CBFVs (cm/s; mean $\pm \mathrm{SD})$ in infants of smoking mothers and in control infants

\begin{tabular}{|c|c|c|c|c|}
\hline & & $\begin{array}{l}\text { Infants of } \\
\text { smoking mothers } \\
(n=41)\end{array}$ & $\begin{array}{l}\text { Control infants } \\
(n=59)\end{array}$ & $P$ \\
\hline \multirow[t]{3}{*}{ ICA: } & $V_{\max }$ & $85.6 \pm 16.0$ & $76.5 \pm 21.0$ & $<0.05$ \\
\hline & $V_{\text {mean }}$ & $46.4 \pm 9.0$ & $39.4 \pm 12.0$ & $<0.01$ \\
\hline & $\mathrm{V}_{\text {ed }}$ & $22.0 \pm 4.6$ & $20.0 \pm 6.0$ & $<0.05$ \\
\hline \multirow[t]{3}{*}{ ACA: } & $\mathrm{V}_{\max }$ & $65.4 \pm 10.7$ & $46.7 \pm 11.5$ & $<0.01$ \\
\hline & $V_{\text {mean }}$ & $35.5 \pm 6.3$ & $25.3 \pm 6.0$ & $<0.01$ \\
\hline & $\mathrm{V}_{\mathrm{ed}}$ & $16.6 \pm 4.1$ & $12.7 \pm 3.9$ & $<0.01$ \\
\hline \multirow[t]{3}{*}{ BA: } & $V_{\max }$ & $67.2 \pm 15.1$ & $54.2 \pm 12.9$ & $<0.01$ \\
\hline & $V_{\text {mean }}$ & $36.8 \pm 8.8$ & $29.5 \pm 7.2$ & $<0.01$ \\
\hline & $V_{\text {ed }}$ & $17.1 \pm 4.3$ & $14.9 \pm 3.7$ & $<0.01$ \\
\hline
\end{tabular}

ICA, Internal carotid artery; ACA, anterior cerebral artery; BA, basilar artery; $\mathrm{V}_{\max }$, maximal systolic flow velocity; $\mathrm{V}_{\text {mean }}$, mean flow velocity; $V_{e d}$, end-diastolic flow velocity

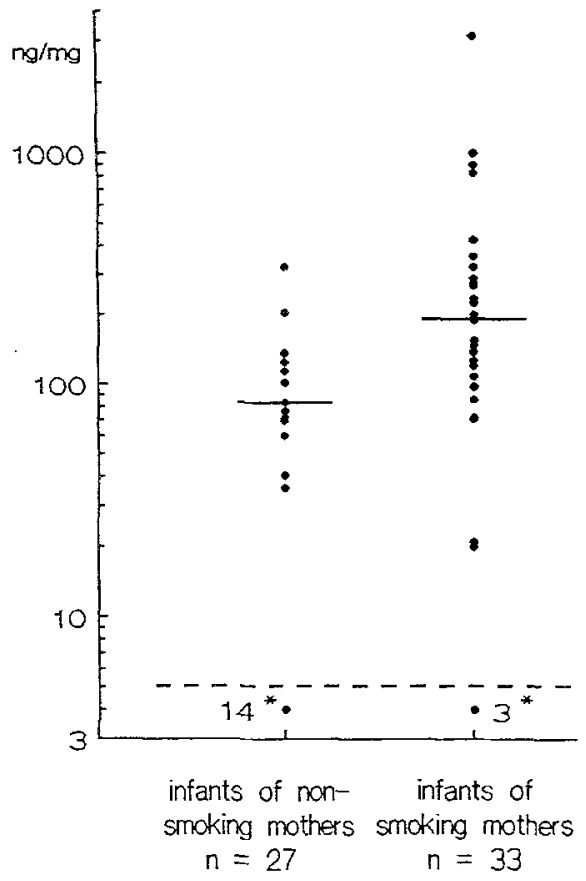

Fig. 1. Urinary cotinine/creatinine excretion ( $\mathrm{ng} / \mathrm{mg}$ ) in newborn infants with and without a maternal smoking history. —, medians; *, number of values below detection limit (-----)

mg creatinine. In the group of control infants, urine samples could be obtained form 27 infants. In 14 of these, cotinine was below the detection limit; in the remaining 13 infants, urinary cotinine excretion ranged from 35.8 to 203.9 , median $83.6 \mathrm{ng} / \mathrm{mg}$ creatinine (Fig. 1).

When only those infants were evaluated whose mothers either had admitted to smoke and whose urinary cotinine excretion was elevated, or whose mothers denied smoking and whose urinary cotinine excretion was negative, the CBFVs of these two groups still differed significantly (data not shown).

Table 2 a shows univariate correlation coefficients between the mean CBFV in the anterior cerebral artery ("ACAmean") and those factors with a possible influence on CBFVs in newborn infants. Table 2 also shows the univariate intercorrelation coefficients between these factors. ACAmean is depicted as an example of the results which we obtained when we analysed the influence of those factors on all nine CBFVs listed in Table 1.

Table $2 \mathrm{~b}$ presents the results of a multivariate regression analysis for ACAmean as the dependent variable and the factors entered as independent variables. The factor "prenatal smoke exposure" has a highly significant predictive value in this statistical model no matter which method of entering variables into the multiple regression model (stepwise, forward selection, backward elimination) was used. Gestational age also had some predictive value but with less influence than smoke exposure as can be estimated from the Beta value given in the Table. For this model, the multiple correlation coefficient $r$ is $0.70 ; r^{2}$, which corresponds to the part of the variance of ACAmean that can be predicted on the basis of the multiple regression model, is 0.49. Apart from "prenatal smoke exposure" and "gestational age", 
Table 2. Univariate correlation coefficients (a) and multivariate regression analysis (b) between ${ }^{a}$ mean CBFV in the anterior cerebral artery (dependent variable) and ${ }^{\mathrm{b}}$ prenatal smoke exposure, ${ }^{\mathrm{c}}$ gestational age, ${ }^{\mathrm{d}}$ birth weight, ${ }^{\mathrm{e}}$ umbilical artery $\mathrm{pH}$, and ${ }^{\mathrm{f}}$ systolic
$\mathrm{BP},{ }^{\mathrm{g}}$ heart rate, and ${ }^{\mathrm{h}}$ age at examination (independent variables). Beta is the standardised partial regression coefficient illustrating the contribution of the corresponding factor to $r$, the multiple regression coefficient

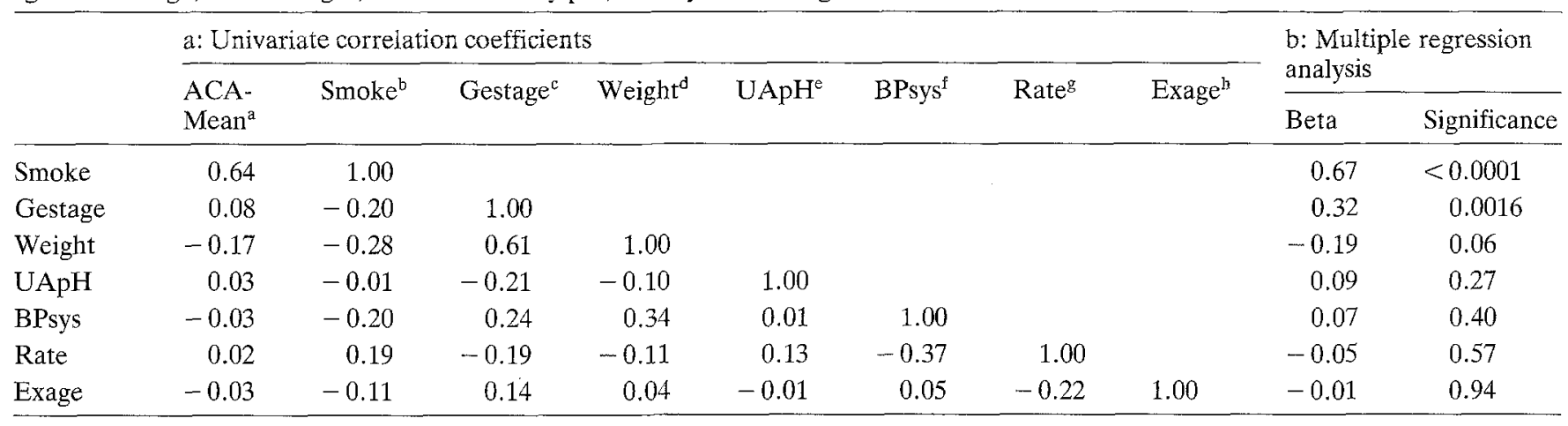

no further variables improved this statistical model significantly. This was also true for the other CBFVs.

\section{Discussion}

CBFVs in newborn infants are influenced by various factors such as birth weight, gestational age, postnatal age, behaviour state, drug administration, and intracranial or cardio-pulmonary abnormalities $[16,18]$. In our study, according to the lower birth weight and gestational age of babies born to smoking mothers compared to control infants, lower CBFVs were to be expected in the former group [7]. However, significantly higher CBFVs were found in smokers' babies.

We did not find factors other than prenatal exposure to tobacco smoke to explain higher CBFVs in the study group. We cannot completely exclude that differences in the arterial $\mathrm{CO}_{2}$ tensions of smoke-exposed and control infants may exist which could influence the CBFVs [18]. However, as we do not know of data reporting an influence of prenatal smoke exposure on $\mathrm{CO}_{2}$ tensions, we did not consider it acceptable to determine arterial blood gases in apparently healthy infants before we knew whether or not there would be any differences in CBFVs at all. The CBFVs in our control infants were in the same range as those observed by others [7]. We tried to confirm prenatal exposure to tobacco smoke in the infants of smoking mothers by determination of their urine cotinine excretion. However, in some of the smokers' babies, we were unable to detect any cotinine excretion, but a cotinine excretion above the detection limit was found in nearly half of 27 control infants. Cotinine excretion varies considerably in smoke-exposed babies [12], partly due to the intensity of exposure and the time interval between exposure and sample collection. Therefore, if a mother did not smoke shortly before and during delivery, the cotinine excretion of her baby may have fallen below the detection limit by the 2nd day of life although the infant had been smoke-exposed during intrauterine life. On the other hand, some mothers of the "control" infants showing cotinine excretion may have smoked themselves or may have been exposed to passive smoking [8]. Nevertheless, the analysis of only those infants with maternal history and laboratory confirmation of smoke exposure or non-exposure, respectively, revealed the same findings as the whole, more heterogeneous study group.

Prenatal Doppler studies $[10,14]$ have demonstrated an acute increase in fetal blood flow velocities associated with elevated maternal BP and heart rate as well as an increase in fetal heart rate immediately after maternal smoking. We do not believe that the differences in CBFVs observed in our study between infants of smoking mothers and controls can be explained by such acute effects of nicotine, catecholamines [17], or carbon monoxide [11], for both nicotine and carbon monoxide have a half-life too short to explain a prolonged influence in infants $20 \mathrm{~h}$ or more after birth. Also, if nicotine were directly responsible for the increased CBFVs, concomitant catecholamine-mediated changes in BP and heart rate should be expected but could not be observed in this study. Therefore we assume that the increased CBFVs represent a prolonged effect of tobacco smoke exposure. Unfortunately, no second measurement could be carried out in this study as most of these apparently healthy infants were discharged home with their mothers on their 3rd or 4th day.

Increased CBFV may indicate changes in the vascular tone in newborn infants of smoking mothers $24-42 \mathrm{~h}$ after birth although the measurement of blood flow ve locities by the Doppler method does not allow differentiation between changes in blood flow and changes in cross-sectional area of the insonated vessel [18]. If we assume that the vascular tone of cerebral arteries is increased after prenatal smoke exposure, this could be explained by alterations of the synthesis of endothelial derived vaso-active factors that play an important role in the regulation of the cerebral circulation [9]. This speculation would be in agreement with the observation of a reduced synthesis of prostacyclin in umbilical arteries from newborns of smoking mothers $[1,6]$.

Further investigations are necessary to evaluate whether the relation between increased CBFVs in newborn infants and prenatal tobacco smoke exposure is not only statistical but can be explained by functional or struc- 
tural alterations of cerebral blood vessels, and how long such alterations persist beyond the first days of life.

Acknowledgements. This work was supported by the Deutsche Forschungsgemeinschaft, SFB 174-A9. The authors thank Mr. N. Wagner for technical assistance, and PD Dr.Dr. W. Hopfenmüller for statistical advice.

\section{References}

1. Ahlsten G, Ewald U, Tuvemu T (1987) Impaired vascular reactivity in newborn infants of smoking mothers. Acta Paediatr Scand 76:248-253

2. Bergman AB, Wiesner LA (1976) Relationship of passive cigarette smoking to sudden infant death syndrome. Pediatrics $58 ; 665-668$

3. Bor M van de, Walther FJ, Sims ME (1990) Increased cerebral blood flow velocity in infants of mothers who abuse cocaine. Pediatrics $85: 733-736$

4. Bulterys MG, Greenland S, Kraus JF (1990) Chronic fetal hypoxia and sudden infant death syndrome: interaction between maternal smoking and low hematocrit during pregnancy. Pediatrics $86: 535-540$

5. Cnattingius S, Haglund B, Meirik O (1988) Cigarette smoke as risk factor for late fetal and early neonatal death. BMJ 279 : 258-261

6. Dadak C, Leithner C, Sinzinger H, Silberbauer K (1981) Diminished prostacyclin formation in umbilical arteries of babies born to women who smoke. Lancet I:94

7. Deeg KH, Rupprecht T (1989) Pulsed Doppler sonographic measurement of normal values for the flow velocities in the intracranial arteries of healthy newborns. Pediatr Radiol 19:7178

8. Greenberg RA, Haley NJ, Etzel R, Loda F (1984) Measuring the exposure of infants to tobacco smoke, nicotine and cotinine in urine and saliva. N Engl J Med 310:1075-1078
9. Leffler CB, Busija DW (1987) Arachidonic acid metabolites and perinatal cerebral hemodynamics. Semin Perinatol 11:3142

10. Lindblad A, Marsal K, Andersson K-E (1988) Effect of nicotine on human fetal blood flow. Obstet Gynecol 72:371-382

11. Longo LD (1979) The biological effects of carbon monoxide on the pregnant woman, fetus, and newborn infant. Am J Obstet Gynecol 129:69-103

12. Luck W, Nau H (1985) Nicotine and cotinine concentration in serum and urine of infants exposed via passive smoking or milk from smoking mothers. J Pediatr 107:816-820

13. Merritt TA (1981) Smoking mothers affect little lives. Am J Dis Child 135: 501-502

14. Morrow RJ, Ritchie JWK, Bull SB (1988) Maternal cigarette smoking: the effects on umbilical and uterine blood flow velocity. Am J Obstet Gynecol 159:1069-1071

15. Naeye RL, Peters EC (1984) Mental development of children whose mothers smoked during pregnancy and effects on the fetus. Obstet Gynecol 64:601-607

16. Perlman JM (1985) Neonatal cerebral blood flow velocity measurement. Clin Perinatol 15:179-193

17. Quigley ME, Sheehan KL, Wilkes MM, Yen SSC (1979) Effects of maternal smoking on circulating catecholamine levels and fetal heart rates. Am J Obstet Gynecol 133:685-689

18. Raju TNK (1991) Cerebral Doppler studies in the fetus and newborn infant. J Pediatr 119:165-174

19. Rantakalio P (1983) A follow-up to the age of 14 of children whose mothers smoked during pregnancy. Acta Paediatr Scand 72:747-753

20. Sexton M, Fox NL, Hebel JR (1990) Prenatal exposure to tobacco: II Effects on cognitive functioning at age three. Int $J$ Epidemiol 19:72-77

21. Sokol JR, Drugan A, Evans M (1990) Substance abuse, smoking in pregnancy. In: Eden RD, Boehm FH (eds) Assessment and care of the fetus, physiological, clinical and medicolegal principles. Prentice - Hall International Inc, East Norwalk, Connecticut, USA, pp 687-691 